\title{
Editorial: Drug Development and Target Discovery in Pulmonary Vascular Diseases
}

\author{
Rui Zhang ${ }^{1}$, Yang-Yang $\mathrm{He}^{2}$ and Zhi-Cheng Jing ${ }^{1,2 *}$ \\ 1 Department of Cardio-Pulmonary Circulation, Shanghai Pulmonary Hospital, Tongji University School of Medicine, \\ Shanghai, China, ${ }^{2}$ Department of Cardiology, Peking Union Medical College Hospital, Chinese Academy of Medical Sciences \\ \& Peking Union Medical College, Beijing, China
}

Keywords: pulmonary vascular disease, pulmonary arterial hypertension, drug development, targeted drug, molecular machine

\section{Editorial on the Research Topic}

Drug Development and Target Discovery in Pulmonary Vascular Diseases

\section{OPEN ACCESS}

Edited and reviewed by: Paolo Montuschi, Catholic University of the Sacred Heart, Italy

*Correspondence: Zhi-Cheng Jing jingzhicheng@vip.163.com

Specialty section:

This article was submitted to Respiratory Pharmacology,

a section of the journal

Frontiers in Pharmacology

Received: 19 February 2020

Accepted: 23 April 2020

Published: 08 May 2020

Citation:

Zhang R, He Y-Y and Jing Z-C (2020)

Editorial: Drug Development and

Target Discovery in Pulmonary

Vascular Diseases.

Front. Pharmacol. 11:660. doi: 10.3389/fphar.2020.00660
Pulmonary vascular disease is a broad category of disorders threatening millions of people in the world, often leading to abnormal blood flow between the lungs and heart. Despite multiple drugs interfering with prostacyclin, endothelin, nitric oxide pathways were available for pulmonary arterial hypertension $(\mathrm{PAH})$ over the past 15 years, it still remained severe and complicated clinical situation (Humbert et al., 2019). The new prevention and treatment strategies for PAH are a major clinical issue as well as a scientific challenge that needed to be addressed urgently. Discovery in pathobiological and cellular mechanisms of pulmonary vascular remodeling will promote the progression of PAH.

We organized the Research Topic entitled "Drug Development and Target Discovery in Pulmonary Vascular Diseases" to discuss and explore new therapeutic targets and mechanism of drug action toward pulmonary vascular disease. The topic covers both basic scientific as well as clinical aspects, consisting 4 original research articles and 1 abstract. Our Research Topic has been well received by the readership of the journal with over 5,000 views and more than 1,000 article downloads.

The feature of PAH is involved in the reduction of prostacyclin synthase expression and disorder of prostacyclin metabolic pathway. The clinical uses of prostacyclin analogues mimic endogenous prostacyclin, which lead to vasodilation and improvement of vascular remodeling (Galiè et al., 2003; Galiè et al., 2016). As a member of the prostacyclin family, iloprost was used for severe PAH and acute right ventricular (RV) failure. The importance for RV physiological changes during acute iloprost inhalation is discussed in the paper of $\mathrm{Li}$ et al. By cardiovascular magnetic resonance imaging, authors showed that acute iloprost inhalation increased RV ejection fraction and RV stroke volume, as well as decreased RV volume in idiopathic PAH and connective tissues disease associated PAH. These observations suggested RV function improvement would be largely attribute to a reduction of afterload by iloprost. This knowledge further added the clinical pharmacological value of iloprost for treatment of PAH.

As a mainstay of contemporary treatment, prostacyclin analogues therapies have been recommended to improve heamodynamics and outcomes in short term trials. (Barst et al., 1996). Beraprost is another chemically stable and orally active prostacyclin analogue. However, their present action mechanisms do not explain the relationship between their receptor subtype and ion 
channels better. Fan et al. demonstrated beraprost was able to down-regulate the expression of pulmonary prostacyclin receptors (IP) and reduce the activity of $\mathrm{O}_{2}$ sensitive potassium ion channels, partly through binding to the prostaglandin receptor E4 (EP4) in both humans and rats. This finding highlights a different binding receptor among iloprost, treprostinil, and beraprost, suggesting a specific E4 agonist may be potential therapeutic values for PAH.

Pulmonary vascular remodeling in PAH not only characterized by different vascular cells accumulation in the pulmonary arterial wall, but also by an exaggerated perivascular infiltration of inflammatory cells (Rabinovitch et al., 2014; Voelkel et al., 2016). Therefore, the possible pathobiological mechanisms of the vascular remodeling and inflammation are need to elucidate urgently. Deng et al. described a selective alpha 7 nicotinic acetylcholine receptor $(\alpha 7 \mathrm{nAChR})$ agonist, PNU-282987, which attenuated progression of monocrotaline (MCT)-induced PAH by downregulating the NLRP3. This article emphasized crosstalk between inflammatory cells and pulmonary artery smooth muscle cells played an important role in the pathogenesis of $\mathrm{PAH}$. The $\alpha 7 \mathrm{nAChR}$ is a ligand-gated ion channel and proved high expression in resident macrophages. The activation of this receptor inhibits the production of inflammatory cytokines, thereby attenuating the local inflammatory response.

In recent years, multiple molecular mechanisms have been explored a lot and new targets may constitute the theoretical basis for future clinical research. A PAH animal model induced by MCT is helpful to study the pathogenesis of $\mathrm{PAH}$ as well as pre-clinical assessment of new therapies (Hill et al., 2017). By this animal model, Zhang et al. identified an active ingredient of Chinese herbal medicine radix glycyrrhizae, $18 \beta$-Glycyrrhetinic acid (18 $\beta-G A)$, which has protective effects against PAH. In a previous study, these authors showed that $18 \beta$-GA played a critical role in antioxidant effect and lung protective activity. The

\section{REFERENCES}

Barst, R. J., Rubin, L. J., Long, W. A., McGoon, M. D., Rich, S., Badesch, D. B., et al. (1996). A comparison of continuous intravenous epoprostenol (prostacyclin) with conventional therapy for primary pulmonary hypertension. N. Engl. J. Med. 334, 296-301. doi: 10.1056/NEJM199602013340504

Galiè, N., Manes, A., and Branzi, A. (2003). Prostanoids for pulmonary arterial hypertension. Am. J. Respir. Med. 2, 123-137. doi: 10.1007/BF03256644

Galiè, N., Humbert, M., Vachiery, J. L., Gibbs, S., Lang, I., Torbicki, A., et al. (2016). 2015 ESC/ERS Guidelines for the diagnosis and treatment of pulmonary hypertension: The Joint Task Force for the Diagnosis and Treatment of Pulmonary Hypertension of the European Society of Cardiology (ESC) and the European Respiratory Society (ERS): Endorsed by: Association for European Paediatric and Congenital Cardiology (AEPC), International Society for Heart and Lung Transplantation (ISHLT). Eur. Heart J. 1, 167-119. doi: 10.1093/eurheartj/ehv317

Hill, N. S., Gillespie, M. N., and McMurtry, I. F. (2017). Fifty years of monocrotaline-induced pulmonary hypertension: what has it meant to the field? Chest 152, 1106-1108. doi: 10.1016/j.chest.2017.10.007

Humbert, M., Guignabert, C., Bonnet, S., Dorfmüller, P., Klinger, J. R., Nicolls, M. R., et al. (2019). Pathology and pathobiology of pulmonary hypertension: state of the present study corroborated the hypothesis that $18 \beta$-GA could reduce oxidative stress condition, mainly down-regulating the expression of both nicotinamide adenine dinucleotide phosphate oxidase-2 (Nox2) and Nox4.

In summary, the above all contributions provide an overview on potential therapeutic targets and discovery of drugs for pulmonary vascular disease. We further advocated the novel scientific knowledge needed to transfer into healthcare interventions better.

\section{AUTHOR CONTRIBUTIONS}

All authors listed have made substantial, direct, and intellectual contribution to the work and approved it for publication.

\section{FUNDING}

This work was supported by grants from the Beijing Natural Science Foundation (7181009), National Natural Science Foundation of China (81630003), 13th Five-Year PlanPrecision Medicine-Key Research and Development ProgramClinical Cohort of Rare Disease (2016YFC0901500), CAMS Innovation Fund for Medical Sciences (2016-I2M-1-002, 2017I2M-BR-02, 2017PT32016).

\section{ACKNOWLEDGMENTS}

The authors would like to thank other topic editors: Lu Hua, XiQi Xu, Kai Sun, Xiao-Jian Wang, Fakhrul Ahsan, and Ji-Wang Chen for their valuable contributions and work of review.

art and research perspectives. Eur. Respir. J. 53, 1801887. doi: 10.1183/ 13993003.01887-2018

Rabinovitch, M., Guignabert, C., Humbert, M., and Nicolls, M. R. (2014). Inflammation and immunity in the pathogenesis of pulmonary arterial hypertension. Circ. Res. 115, 165-175. doi: 10.1161/CIRCRESAHA.113. 301141

Voelkel, N. F., Tamosiuniene, R., and Nicolls, M. R. (2016). Challenges and opportunities in treating inflammation associated with pulmonary hypertension. Expert. Rev. Cardiovasc. Ther. 14, 939-951. doi: 10.1080/ 14779072.2016.1180976

Conflict of Interest: The authors declare that the research was conducted in the absence of any commercial or financial relationships that could be construed as a potential conflict of interest.

Copyright (C) 2020 Zhang, He and Jing. This is an open-access article distributed under the terms of the Creative Commons Attribution License (CC BY). The use, distribution or reproduction in other forums is permitted, provided the original author(s) and the copyright owner(s) are credited and that the original publication in this journal is cited, in accordance with accepted academic practice. No use, distribution or reproduction is permitted which does not comply with these terms. 